\title{
Correction to: Opposite Roles of NT-3 and BDNF in Synaptic Remodeling of the Inner Ear Induced by Electrical Stimulation
}

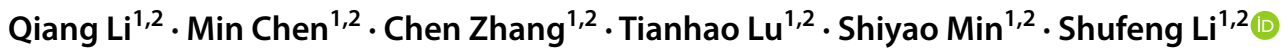

Published online: 25 August 2020

○) Springer Science+Business Media, LLC, part of Springer Nature 2020

\section{Correction to: Cellular and Molecular Neurobiology https://doi.org/10.1007/s10571-020-00935-x}

The original version of this article unfortunately contained an error in Fig. 9.
In article, the image of Figure 8 was inadvertently repeated in place of Fig. 9 instead of placing the appropriate figure. However, the caption of Fig. 9 is correct.

The correct Fig. 9 is presented here.

The original article has been corrected.

2 NHC Key Laboratory of Hearing Medicine (Fudan University), Shanghai, China 

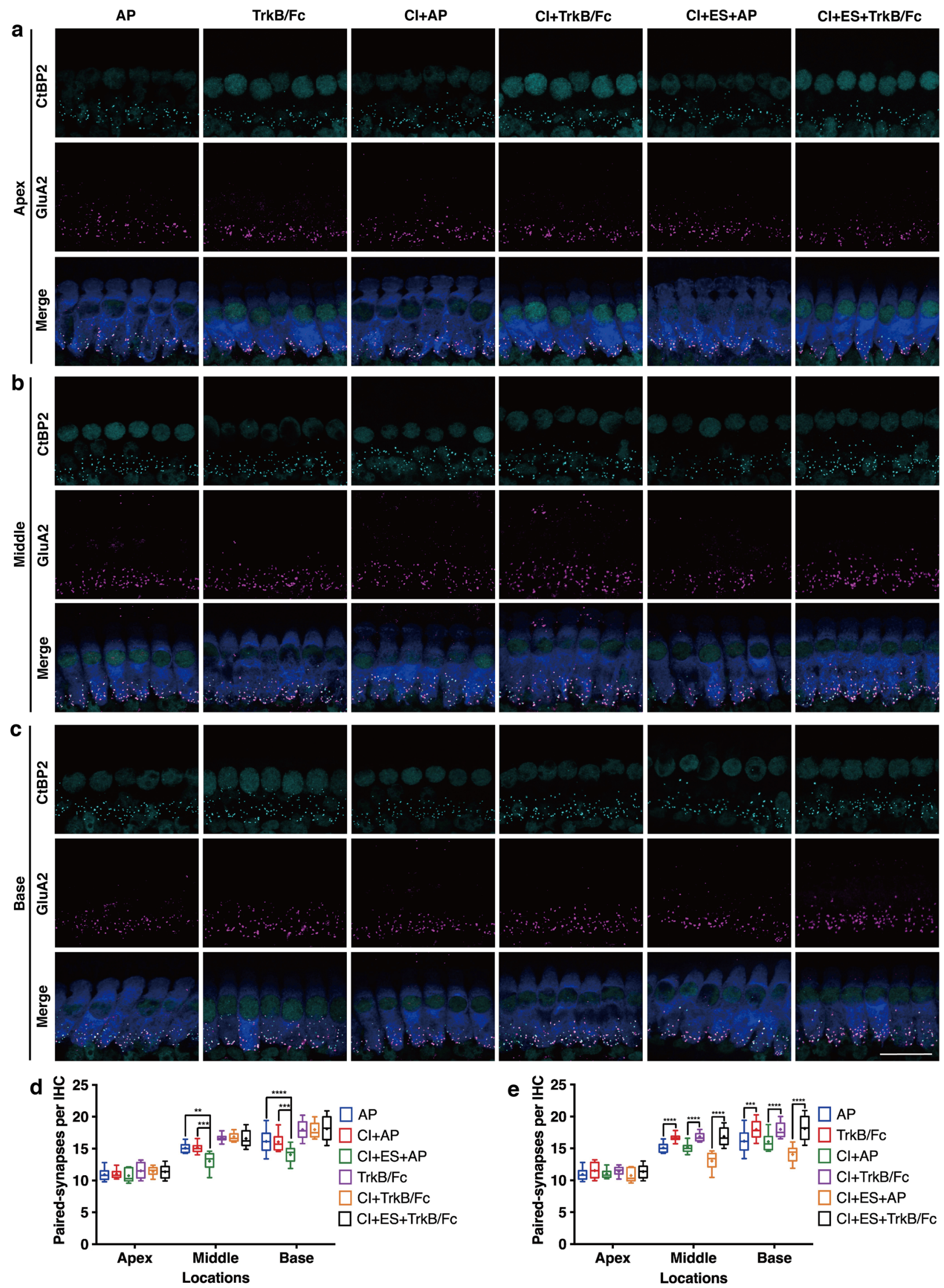
४Fig. 9 The changes of IHC synapses following intracochlear perfusion of exogenous $\mathrm{TrkB} / \mathrm{Fc}$. a-c Immunostaining for presynaptic ribbons, postsynaptic patches, and IHCs at apical (a), middle (b) and basal (c) parts. IHCs (blue, Myosin 7a) were arranged neatly and compactly. Presynaptic ribbons (cyan, $\mathrm{CtBP} 2$ ) and postsynaptic patches (magenta, GluA2) were arranged in pairs at the bottom of the IHCs. The scale bar in $\mathbf{c}$ applies to all panels $(\mathbf{a}-\mathbf{c})$ and represents 20 microns. d Quantity of paired-synapses per IHC under different conditions. One-way ANOVA with post hoc Bonferroni correction or Kruskai- Wallis test with post hoc Dunn test was performed, $n=5$ for each group. e Comparisons of paired synaptic counts between AP and TrkB/Fc perfused cochlea. Two-tailed unpaired student's $t$ test or Mann-Whitney test was conducted, $n=5$ for each group. AP, the ears only perfused with artificial perilymph; TrkB/Fc, the cochlea only perfused with $\mathrm{TrkB} / \mathrm{Fc}$; CI+AP, the cochlea perfused with artificial perilymph and implanted with electrodes; $\mathrm{CI}+\mathrm{TrkB} / \mathrm{Fc}$, the cochlea perfused with $\mathrm{TrkB} / \mathrm{Fc}$ and implanted with electrodes; $\mathrm{CI}+\mathrm{ES}+\mathrm{AP}$, the cochlea perfused with artificial perilymph, implanted with electrodes, and stimulated with electric pulses; CI+ES+TrkB/Fc, the cochlea perfused with $\mathrm{TrkB} / \mathrm{Fc}$, implanted with electrodes, and stimulated with electric pulses. ${ }^{*} P<0.01$, *** $P<0.001$, **** $P<0.0001$
Publisher's Note Springer Nature remains neutral with regard to jurisdictional claims in published maps and institutional affiliations. 